\title{
Contributions to improving the design of a logistics equipment, using artificial neural networks
}

\author{
Daniel - Constantin ANGHEL*, Maria Mihaela ISTRATE \\ University of Pitesti, Pitesti, Romania \\ ${ }^{*}$ Corresponding author e-mail: daniel.anghel@upit.ro \\ Article history \\ Received 20.06.2019 \\ Accepted 15.08.2019 \\ DOI https://doi.org/10.26825/bup.ar.2019.001
}

\begin{abstract}
This paper deals with aspects related to the improvement of the design of a logistics equipment (conveyor belt).

Conveyor belts are very important logistics equipment within the logistics systems and aim at the controlled and safe transport of products, requiring as little as possible the human operator and guaranteeing their safety at the workplace.

The necessity of the study derived from the fact that in the industry there are different situations when the conveyors have to be customized, depending on the activity carried out. In addition, a flexible production line requires "flexible" conveyors, that is to say adaptable to the required situations: transport capacity, ground band height, control panel layout, special conditions (smoke, dust, humidity ... etc).

In order to establish the ergonomic design parameters, we applied concepts from Value Analysis and RULA analysis. The simulation parameters were used to simulate an Artificial Neural Network, which, after training, would be able to provide design requirements for new situations.
\end{abstract}

Keywords: design process, Artificial Neural Networks, Functional Analysis

\section{Introduction}

In order to establish the ergonomic design parameters, we applied concepts from Value Analysis and RULA analysis. The simulation parameters were used to simulate an Artificial Neural Network, which, after training, would be able to provide design requirements for new situations.

\section{Analysis of the conveyor belt with a view to establishing the ergonomic parameters}

This chapter presents the analysis of the product "conveyor belt" with the help of Functional Analysis [YAN 01] tools and a systemic analysis to establish the operator's functions within the HumanMachine-Environment system [MAN 10].

To perform the Functional Analysis, we used "MicroOutils AF" software, Figure 1.

To highlight the functions that man can perform in this system we will consider a job in a company, that of a design engineer within a study office. The designer receives information from various sources: technical documentation received by e-mail, such as computer files, certain requirements 
expressed by telephone or directly by a beneficiary or partner, existing prescriptions in the specialized literature, etc.

Based on this information and taking into account also a number of other factors (experience in the field, complexity of the project, costs, deadlines, etc.) the designer will make a series of decisions for the fulfillment of his tasks. Its mode of action will be based on the decisions taken.

Thus, within this system, man can perform three types of functions, Figure 2:

- receiving information;

- information processing;

- action.

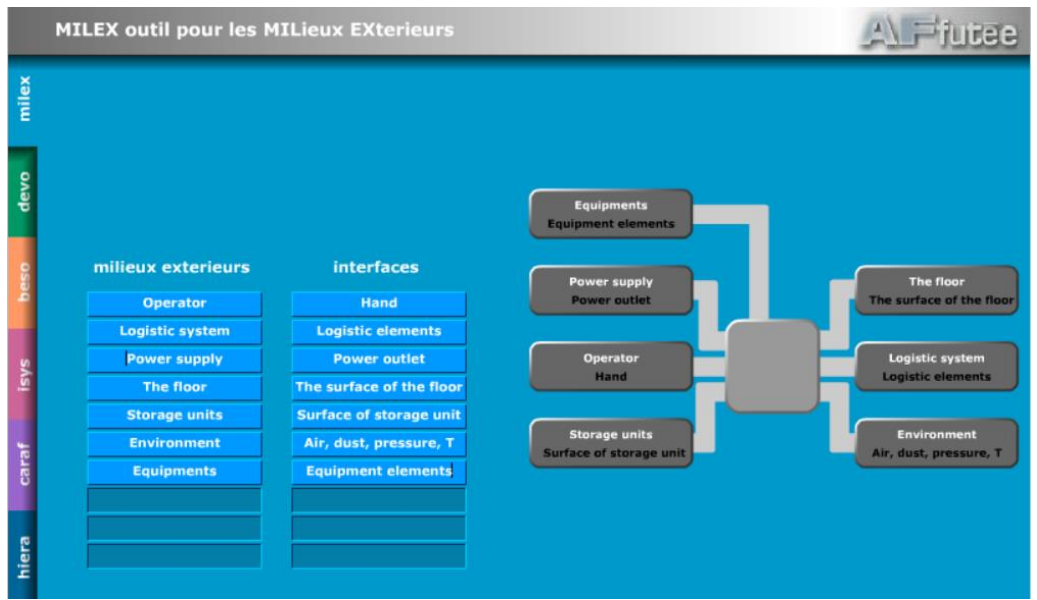

Figure 1. Identification of the External Elements of the product with the help of Functional Analysis

The function of receiving information allows the human being to receive a series of information necessary to perform the tasks. This information, regardless of the source from which it comes, must be in a format that one can recognize and understand.

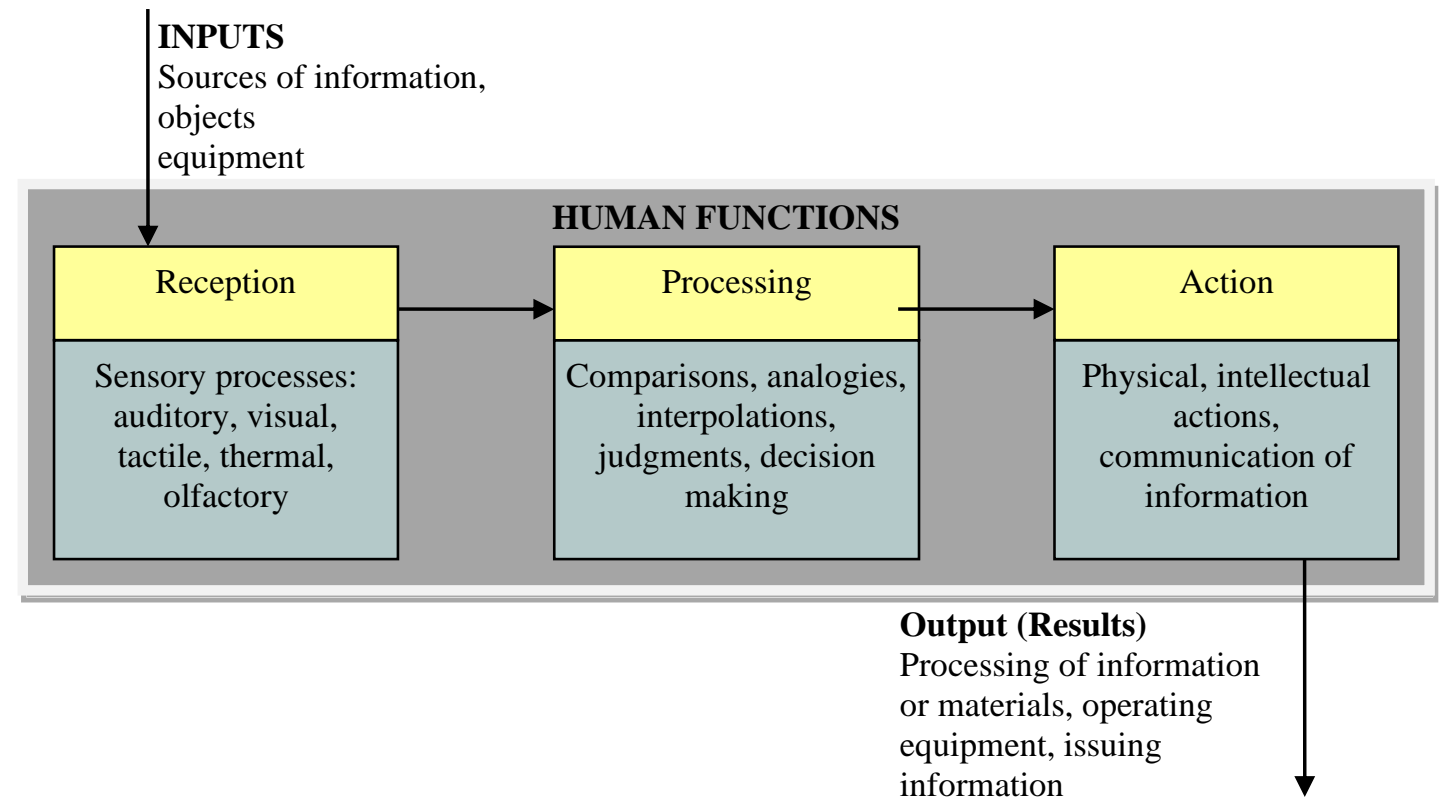

Figure 2. Human functions within the Human-Machine-Environment system

Thus, equipment designers have the possibility to choose the sensory mode by which to receive information (visual, auditory, tactile, etc.). However, it must be taken into account the advantage that one sensory modality has over another and the existence of other equipment that already requires some sensory channels.

The function of information processing helps the person to understand the information for the purpose of making decisions. During the information processing stage, the person performs a series of mental 
operations (analogies, calculations, judgments, evaluations) that have the purpose of making a decision.

The way of processing information and making decisions depends on the type of tasks performed by the man.

If the man is involved in unpredictable tasks, his intellectual abilities are much more demanded than the previous case. Man is constantly receiving new information; he has to process it in different ways. Thus, for the fulfilment of his task, an important role has him: professional experience, human skills and his professional training.

The human action function manifests itself in the form of its interactions with machines, equipment, tools, etc. Thus, when designing machines (machine tools, presses, computers, machines, etc.), tools and equipment must be taken into account the human factor (his motor capabilities, skills, experience, etc.), the machine (to suit human abilities) and the factor environment (the impact of the machine on the working environment).

Taking into account the characteristics presented and the elements that come into interaction with the conveyor, the parameters that will be established for its ergonomic design are the following:

P1. accessibility at the control panel;

P2. the possibility of adjusting the height of the conveyor;

P3. crushing risk;

P4. noise level;

P5. possibility to adjust the speed of the band;

P6. electrocution risk;

P7. simple integration in the logistics system;

P8. easy maintenance;

P9. side protection for storage units;

P10. flexible structure.

We specify the following: normally, we should have given each criterion a certain importance, resulting from a careful analysis. However, the use of neural networks saves us from this, the network learning from examples.

Parameter values can be: -2 Very Low; -1 Low; 0 Acceptable; 1 Good; 2 Very Good.

Considering the aspects presented, a questionnaire has been established. This questionnaire will be distributed to the conveyor operators for the purpose of completing them.

EVALUATION QUESTIONNAIRE
Conveyor parameters
\begin{tabular}{|l|l|l|l|l|l|}
\hline & -2 & -1 & 0 & 1 & 2 \\
\hline P1. accessibility at the control panel; & & & & & \\
\hline P2. the possibility of adjusting the height of the conveyor; & & & & & \\
\hline P3. crushing risk; & & & & & \\
\hline P4. noise level; & & & & & \\
\hline P5. possibility to adjust the speed of the band; & & & & & \\
\hline P6. electrocution risk; & & & & & \\
\hline P7. simple integration in the logistics system; & & & & & \\
\hline P8. easy maintenance; & & & & & \\
\hline P9. side protection for storage units; & & & & \\
\hline P10. flexible structure. & & & & & \\
\hline
\end{tabular}

\section{Conveyor quotation}

\begin{tabular}{|l|l|l|l|l|l|}
\hline & -2 & -1 & 0 & 1 & 2 \\
\hline Quotation & & & & & \\
\hline
\end{tabular}

\section{Description}

\begin{tabular}{|r|r|r|r|r|}
\hline-2 & -1 & 0 & 1 & 2 \\
\hline Very low & low & medium & high & Very high \\
\hline
\end{tabular}

Mark the answer you want with "x".

Figure 3. Evaluation questionnaire 
The distribution of questionnaires was done by a person who is not part of the hierarchy in order not to influence the decisions of the operators.

In order to avoid as much as possible, the external influence factors, the questionnaires were distributed in different periods throughout a calendar month.

In Figure 3 the evaluation questionnaire is presented.

\section{Ergonomic analysis of logistics equipment with artificial neural networks}

This section presents the analysis using Artificial Neural Networks.

\subsection{Training and testing of the neural network}

The choice of parameters was made based on the analysis of conveyors, by placing a virtual dummy in Catia V5, in the ergonomic module, with the RULA method and of existing conveyors in production and by questioning their operators.

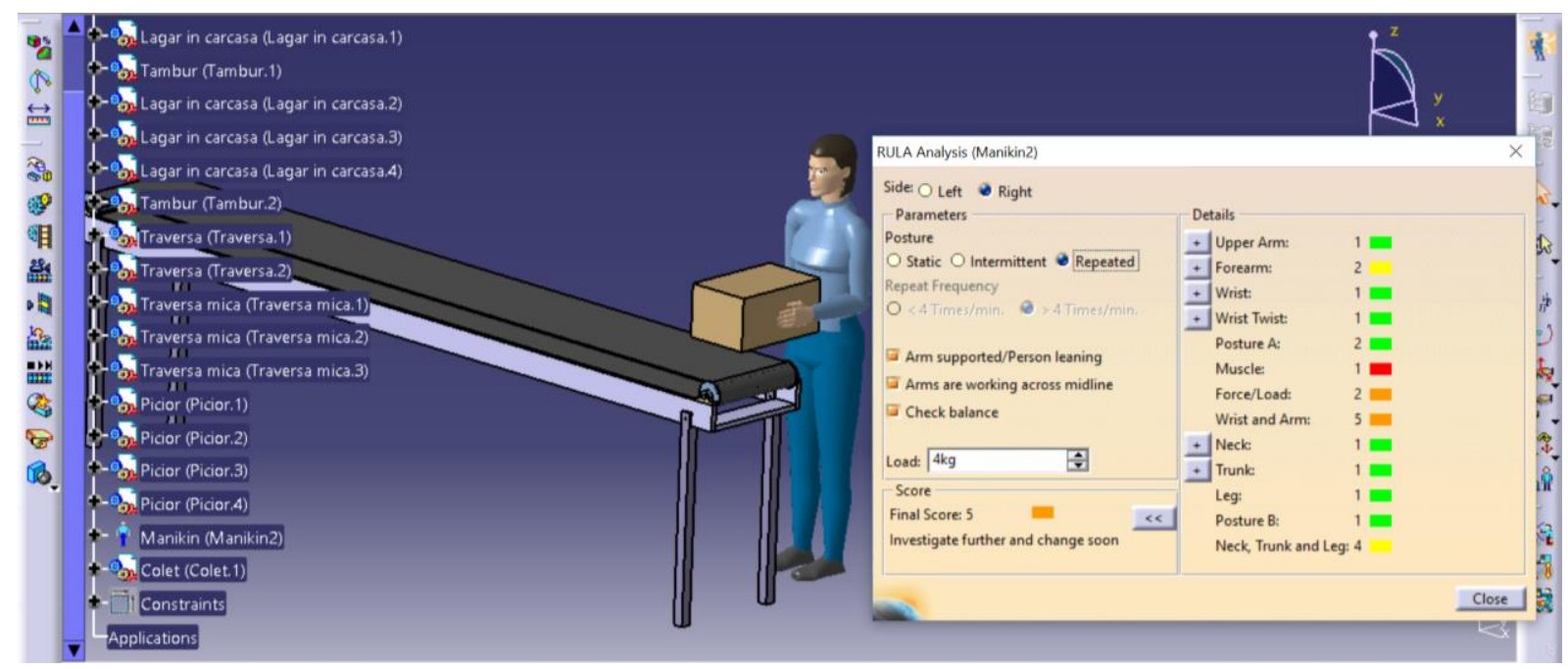

Figure 4. RULA analysis, repeated load, $4 \mathrm{~kg}$, arms along the median line with support

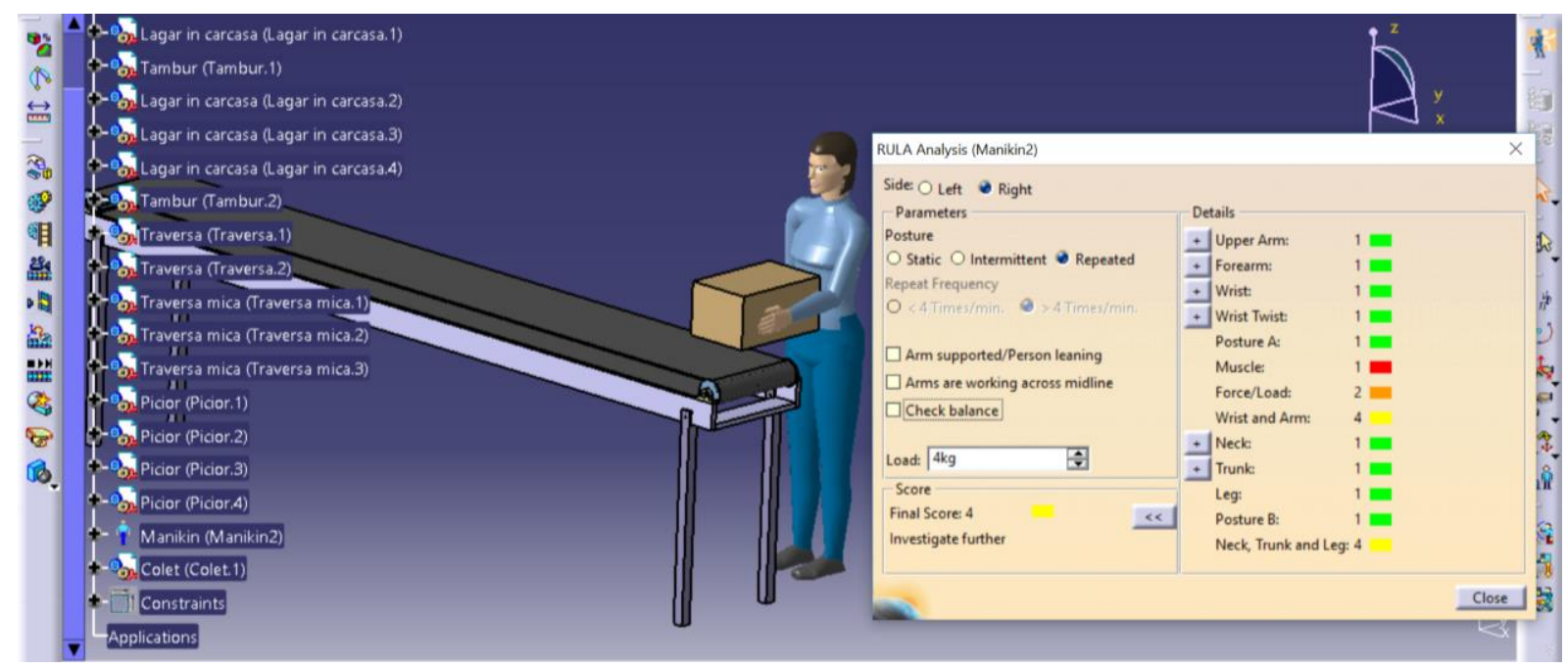

Figure 5. RULA analysis, repeated load, 4kg, arms without support

The parameters chosen for the training and testing of the network are presented in table 1 . 
Table 1. RNA training / testing parameters (extract)

\begin{tabular}{|c|c|c|c|c|c|c|c|c|c|c|c|c|c|c|}
\hline Parameter & \multicolumn{12}{|c|}{ Training } & \multicolumn{2}{|c|}{ Testing } \\
\hline $\mathrm{P} 1$ & 1 & 1 & 0 & 0 & 1 & 1 & 1 & 0 & 0 & 0 & 0 & 0 & 2 & 0 \\
\hline $\mathrm{P} 2$ & 1 & 2 & 1 & 2 & 1 & 2 & 0 & 1 & 1 & 0 & -1 & 0 & 1 & 0 \\
\hline P3 & 2 & 1 & 1 & 1 & 1 & 1 & 1 & -1 & -2 & -2 & -2 & 1 & 1 & 0 \\
\hline P4 & 2 & 0 & 0 & 2 & -2 & 1 & 2 & 1 & 1 & 1 & 1 & 1 & 0 & 1 \\
\hline $\mathrm{P} 5$ & 1 & 0 & 2 & 1 & -2 & 1 & 1 & 2 & 1 & 1 & 1 & -2 & 1 & -1 \\
\hline $\mathrm{P} 6$ & 1 & 0 & 0 & 0 & 0 & 0 & 0 & 1 & 1 & 1 & 2 & -2 & 2 & 1 \\
\hline P7 & 0 & 1 & 1 & 0 & -1 & 0 & 1 & 1 & 1 & 1 & 2 & -2 & 1 & 2 \\
\hline P8 & 1 & -1 & -2 & -2 & -2 & 1 & 1 & 1 & 1 & 1 & 2 & 0 & 0 & 1 \\
\hline P9 & 2 & 1 & 1 & 1 & -1 & 1 & 1 & 0 & 0 & 0 & 2 & 1 & 1 & 1 \\
\hline $\mathrm{P} 10$ & 1 & 2 & 1 & 1 & -1 & -2 & 1 & 2 & 1 & 2 & 2 & 0 & 1 & 1 \\
\hline Equipment rating & 1 & 1 & 1 & 1 & -2 & 2 & 1 & 1 & 2 & 2 & 1 & -1 & 1 & 1 \\
\hline
\end{tabular}

The data is imported into the MATLAB Toolbox.

For the input sizes we created a matrix of 10x40 (Conveyor parameters), and for the output sizes a matrix (vector) of 1x40 (Equipment rating).

In order to train, test and validate the network, we divided the 40 data sets into 3 categories: $70 \%$ for training (28 data sets) $15 \%$ for testing and $15 \%$ for validation.

The Neural Network Fitting Tool has the possibility to randomly distribute the data sets in the three categories.

The network was trained using the "Levenberg-Marquardt" backpropagation algorithm [ANG 19], Figure 6. This process is an iterative one, in this case, to train the network a number of 555 iterations was required.

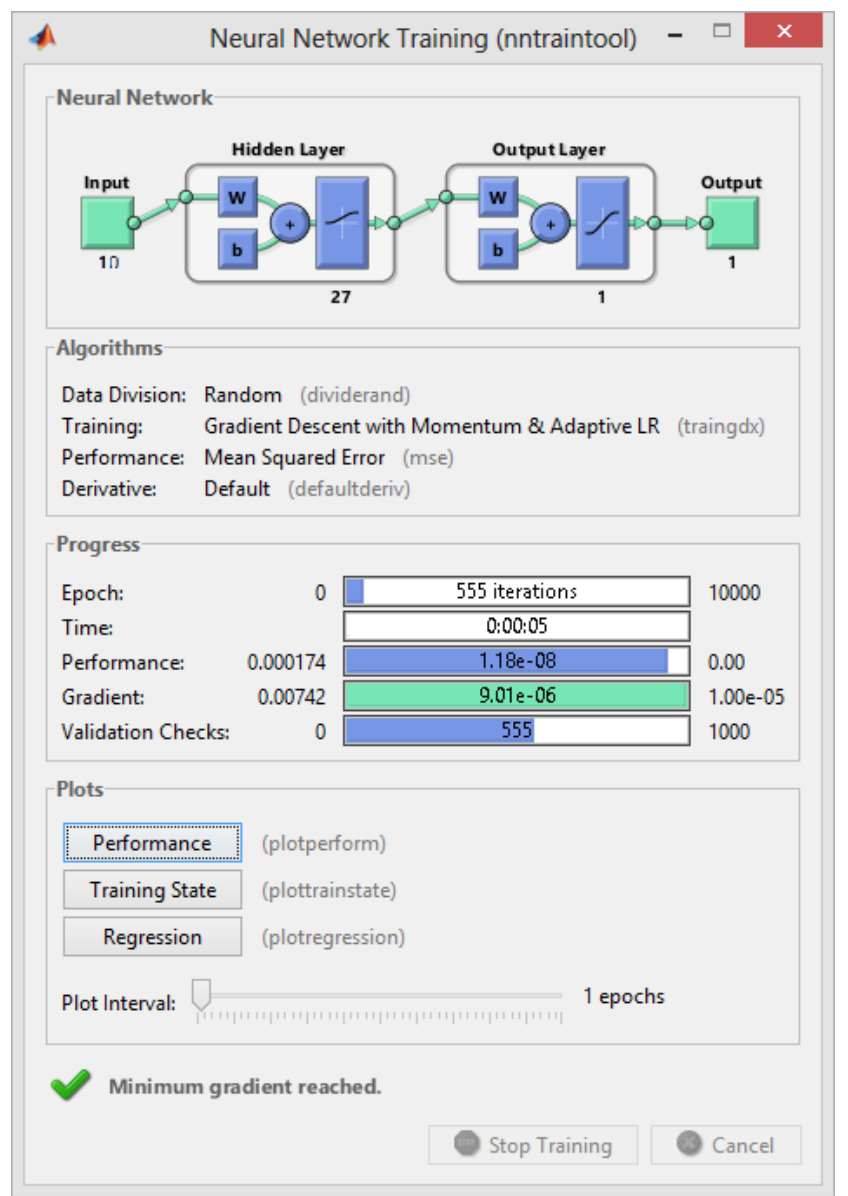

Figure 6. Training the neural network 
When the training process is considered completed, the results are displayed in the Train Network window. The values for $\mathrm{R}$ and the mean square error are displayed.

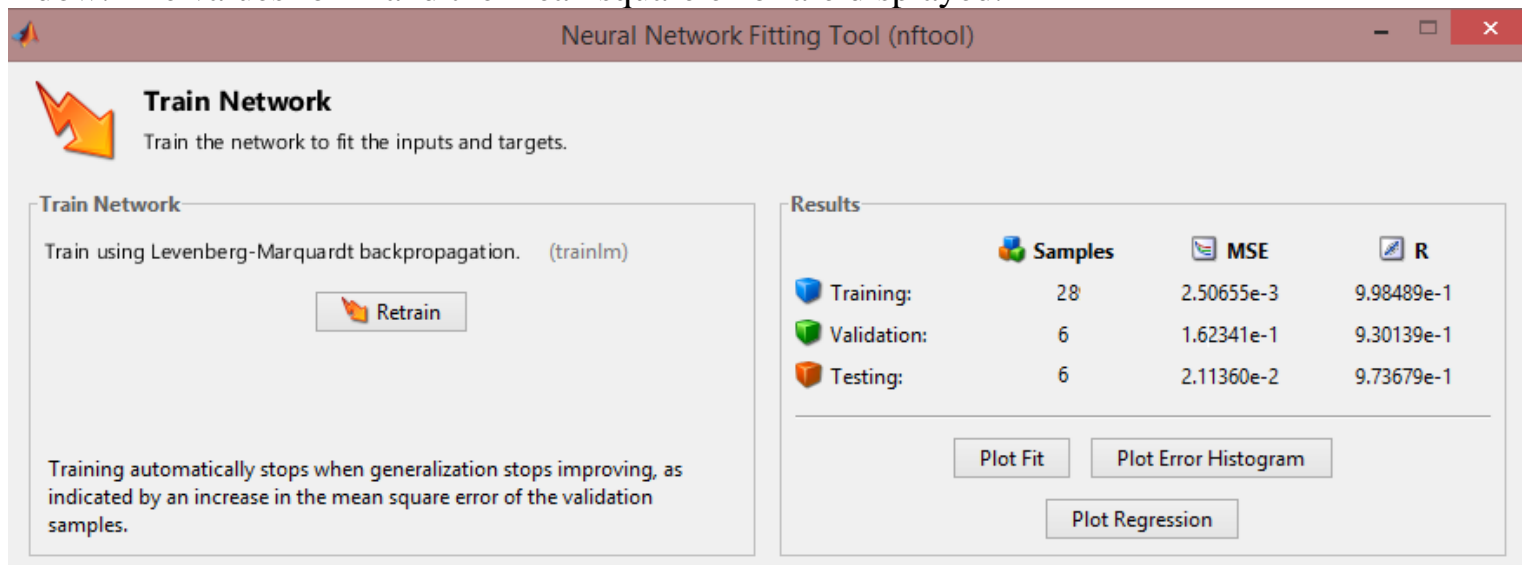

Figure 7. The results of network training, testing and validation

After training the network, it is used by the designer to ask for advice in order to "simulate" some proposed solutions, different from those in practice.

Thus, we will introduce a new set of input data, corresponding to the conveyor to be designed and thus we will be able to find its quotation given by the network.

Table 2. Parameters of the designed conveyor and RNA quotation

\begin{tabular}{|l|l|}
\hline Parameter & \\
\hline P1 & 1 \\
\hline P2 & 1 \\
\hline P3 & 2 \\
\hline P4 & 0 \\
\hline P5 & 1 \\
\hline P6 & 2 \\
\hline P7 & 1 \\
\hline P8 & 2 \\
\hline P9 & 0 \\
\hline P10 & 1 \\
\hline Equipment rating & 1 \\
\hline
\end{tabular}

\section{Conclusions}

The combined usage of Functional Analysis and Artificial Neural Networks allows us to realize a rapid and relevant study. By applying the methods of functional analysis, the relationships of the equipment with the environment are determined, and then, after collecting the data from the questionnaires, the database will be used to train, test and validate the neural network. This network can be used by conveyor designers to create new conveyors, better adapted to the human operator, but also by ergonomics specialists to detect non-compliant equipment.

\section{References}

[1] Anghel, D. C., Rizea, A. D., Iordache, D. M., \& Beșliu-Gherghescu, M. L. (2019, October). Studies on the influence of design parameters on the behaviour at shock of 3D-printed components fabricated by fused deposition modelling. In IOP Conference Series: Materials Science and Engineering (Vol. 564, No. 1, p. 012014). IOP Publishing.

[2] Manolescu, A., Lefter, V., Deaconu, A., \& Marinaş, C. (2010). Ergonomie. Editura Economică, 333-338;

[3] Yannou B. , Hajsalem S., Comparaison des apports de l'Analyse de la Valeur et de la méthode SPEC sur un cas industriel, 2001, PRIMECA'2001 : Septième Colloque sur la Conception Mécanique Intégrée, La Plagne, France, 422-432. 